\title{
Lipooligosaccharide structure is an important determinant in the resistance of Neisseria gonorrhoeae to antimicrobial agents of innate host defense
}

\author{
Jacqueline T. Balthazar ${ }^{1,2}$, Asiya Gusa ${ }^{2}$, Larry E. Martin ${ }^{1,3}$, Biswa Choudhury ${ }^{4+}$, Russell Carlson ${ }^{4}$ and \\ William M. Shafer ${ }^{1,2 *}$ \\ 1 Laboratories of Bacterial Pathogenesis, VA Medical Research Service, VA Medical Center, Decatur, GA, USA \\ 2 Department of Microbiology and Immunology, Emory University School of Medicine, Atlanta, GA, USA \\ 3 Department of Medicine, Emory University School of Medicine, Atlanta, GA, USA \\ ${ }^{4}$ Complex Carbohydrate Research Center, University of Georgia, Athens, GA, USA
}

\section{Edited by:}

Cynthia N. Cornelissen, Virginia

Commonwealth University School of

Medicine, USA

Reviewed by:

Sanjay Ram, University of

Massachusetts Medical Center, USA

Christopher Davies, Medical University

of South Carolina, USA

*Correspondence:

William M. Shafer, Department of

Microbiology and Immunology, Emory

University School of Medicine, 1510

Clifton Road, Atlanta, GA 30322, USA.

e-mail:wshafer@emory.edu

\section{${ }^{\dagger}$ Current address:}

Biswa Choudhury, Glycotechnology

Core Resource, University of San

Diego, La Jolla, CA 92093-0687, USA.
The strict human pathogen Neisseria gonorrhoeae has caused the sexually transmitted infection termed gonorrhea for thousands of years. Over the millennia, the gonococcus has likely evolved mechanisms to evade host defense systems that operate on the genital mucosal surfaces in both males and females. Past research has shown that the presence or modification of certain cell envelope structures can significantly impact levels of gonococcal susceptibility to host-derived antimicrobial compounds that bathe genital mucosal surfaces and participate in innate host defense against invading pathogens. In order to facilitate the identification of gonococcal genes that are important in determining levels of bacterial susceptibility to mediators of innate host defense, we used the Himar I mariner in vitro mutagenesis system to construct a transposon insertion library in strain F62. As proof of principle that this strategy would be suitable for this purpose, we screened the library for mutants expressing decreased susceptibility to the bacteriolytic action of normal human serum (NHS). We found that a transposon insertion in the lgtD gene, which encodes an $\mathrm{N}$-acetylgalactosamine transferase involved in the extension of the $\alpha$-chain of lipooligosaccharide (LOS), could confer decreased susceptibility of strain F62 to complement-mediated killing by NHS. By complementation and chemical analyses, we demonstrated both linkage of the transposon insertion to the NHS-resistance phenotype and chemical changes in LOS structure that resulted from loss of LgtD production. Further truncation of the LOS $\alpha$-chain or loss of phosphoethanolamine (PEA) from the lipid A region of LOS also impacted levels of NHS-resistance. PEA decoration of lipid A also increased gonococcal resistance to the model cationic antimicrobial polymyxin B. Taken together, we conclude that the Himar I mariner in vitro mutagenesis procedure can facilitate studies on structures involved in gonococcal pathogenesis.

Keywords: Neisseria gonorrhoeae, mutagenesis, complement, antimicrobial, peptide, resistance, lipooligosaccharide

\section{INTRODUCTION}

Neisseria gonorrhoeae is a strict human pathogen that has caused the sexually transmitted disease gonorrhea for thousands of years (Sparling et al., 1990). Over the millennia, gonococci have developed a number of mechanisms to escape both innate and adaptive immune responses of the human host, which likely explains why immunity to re-infection does not occur. The ability of gonococci to alter the structure of a number of surface antigens or to variably produce them or to express surface structures that are similar to host antigens ("molecular mimicry") have been invoked (Sparling et al., 1990) as mechanisms by which this pathogen evades both innate and adaptive host defense systems.

We are interested in the genetic basis for how gonococci can evade antimicrobial agents that it encounters during infection of mucosal surfaces or when growing in the bloodstream. In this respect, our previous work showed that the MtrC-MtrD-MtrE efflux pump can export host-derived antimicrobial agents such as cationic antimicrobial peptides (CAPs; Shafer et al., 1998), as well as certain classical antibiotics (Veal et al., 2002). In addition to energy-dependent efflux, gonococcal resistance to CAPs has been linked to the decoration of lipid A by phosphoethanolamine (PEA; Lewis et al., 2009), which likely interferes with the ability of CAPs to bind to negatively charged groups on the bacterial surface.

In order to identify additional gonococcal determinants important for bacterial resistance to host-derived antimicrobials, we constructed a Himar I mariner transposon (Pelicic et al., 2000) mutant library of strain F62. We screened this library for mutants expressing decreased susceptibility to normal human serum (NHS) because the ability of gonococci to escape such killing is of likely importance in its capacity to proliferate if it enters the blood stream or if it encounters lethal levels of complement components and natural antibody at mucosal surfaces (Schoolnik et al., 1976; Rice et al., 1980; Rice, 1989; Shafer et al., 1982, 1984) Since NHS killing of strain F62 (and other NHS-sensitive gonococci) can be 
potent, we hypothesized that it could be used to directly select transposon-facilitated NHS-resistant mutants in a library of susceptible bacteria. NHS killing of gonococci is principally mediated by an antibody-dependent (IgM) mechanism involving the classical complement pathway (CCP; Shafer et al., 1982, 1984, 2002; Rice, 1989). While most strains of gonococci are NHS-sensitive, some strains can display a stable NHS-resistance phenotype and they are more frequently isolated from patients with disseminated gonococcal infection (DGI; Schoolnik et al., 1976, Rice et al., 1980). Stable NHS-resistance expressed by these gonococci has been associated with the production of a certain serotype of the major outer membrane porin (Por1A vs. Por1B; Cannon et al., 1983; Rice, 1989; Ram et al., 1998; Ram, 2001), production of a 3.6-kDa lipooligosaccharide (LOS; Schneider et al., 1985; Shafer et al., 2002), and decoration of lipid A by PEA (Lewis et al., 2009).

In the present study we obtained additional evidence that LOS structure is important in determining whether gonococci can resist killing by NHS. This was surmised because a stable Himar I mariner transposon insertion in a gene (lgtD) encoding a glycosyltransferase that adds $N$-acetyl-galactosamine (GalNAc) to the terminal galactose in the LOS $\alpha$-chain rendered strain F62 less susceptible to such killing. $\lg t D$ is a phase variable gene within the $\lg t A B C D E$ operon that switches from phase-on to phase-off (and vice versa) at high frequencies due to a homopolymeric repeat within the coding sequence (Gotschlich, 1994). This mutant encouraged us to more closely evaluate the role of LOS in determining the ability of strain F62 to resist host defensive agents. Importantly, this work shows that the Himar I mariner transposon mutagenesis procedure, previously employed with success in Neisseria meningitidis (Pelicic et al., 2000), can be used to study mechanisms of pathogenesis in gonococci.

\section{MATERIALS AND METHODS BACTERIAL STRAINS, CULTURE CONDITIONS, AND BACTERICIDAL ASSAYS}

Neisseria gonorrhoeae strain F62, which was kindly provided by S. Morse (Centers for Disease Control and Prevention, Atlanta, GA, USA), was the primary strain employed in this study and was cultured from a lyophilized stock prepared by Kellogg in 1962 (Kellogg et al., 1963). It was routinely cultured on GCB agar plates containing defined supplements I and II (Shafer et al., 1982) in 3.8\% (v/v) $\mathrm{CO}_{2}$ at $37^{\circ} \mathrm{C}$ as a piliated $(\mathrm{P}+)$, opacity-negative $(\mathrm{Opa}-)$ variant for transformation experiments and as a $\mathrm{P}-\mathrm{Opa}$ - variant for serum bactericidal assays (SBA). The SBA employed pooled NHS and heat-inactivated NHS ( $\triangle$ NHS), as described previously (Shafer et al., 1982). Gonococci were incubated with serum samples for $45 \mathrm{~min}$ at $37^{\circ} \mathrm{C}$ prior to plating onto GCB agar. In this assay, NHS-resistance is defined as $>30 \%$ survival calculated on the input $\left(10^{5} \mathrm{cfu}\right)$ of gonococci in the reaction mixture. Naturally NHS-resistant strain FA19 was used as a control since it can survive in 50\% (v/v) NHS concentrations (Shafer et al., 1982, 2002). All assays were repeated in triplicate and the data were calculated as average values \pm SD. The significance of the results was determined by Student's $t$-test. Serum was obtained by venipuncture from healthy volunteers after written informed consent and the collection of such serum was approved by the Institutional Review Board of Emory University. The agar dilution method for determining the minimal inhibitory concentration (MIC) for polymyxin B (PMB) was as described previously (Lewis et al., 2009).

\section{CONSTRUCTION AND ANALYSIS OF A HIMAR I MARINER MUTANT LIBRARY OF STRAIN F62 AND ISOLATION AND MOLECULAR CHARACTERIZATION OF AN NHS-RESISTANT MUTANT}

Himar I transposase was purified (Pelicic et al., 2000) and used for in vitro transposition reactions with bulk DNA purified from strain F62, as described previously (Alexander et al., 2006). The transposition reaction mixture was used to transform piliated strain F62, as described previously (Gunn and Stein, 1996), and transformants were selected on GCB agar plates containing $100 \mu \mathrm{g}$ of kanamycin $(\mathrm{Kan}) / \mathrm{mL}$. This procedure was repeated until a total of 10,000 transformants were obtained. All transformants were passed on GCB agar containing Kan and then resuspended in $100 \mu \mathrm{l}$ of freezing media in 96 well microtiter plates and stored at $-80^{\circ} \mathrm{C}$. A pool of the library was also made and similarly stored. In order to determine whether random insertion of the Himar I transposon was achieved, DNA was prepared from 20 mutants. The DNA preparations were digested with ClaI and subjected to agarose gel electrophoresis followed by Southern hybridization analysis (Southern, 1975) using the $a p h A-3$ gene as a transposon-specific probe (Pelicic et al., 2000). The results showed that the DNA from these mutants had a single insertion and that the insertion was in distinct ClaI fragments (data not presented).

In order to isolate Himar I mutants of F62 expressing decreased susceptibility to NHS, the library was grown overnight on GCB agar plates containing $\mathrm{Kan}$ at $37^{\circ} \mathrm{C}$ under $3.8 \%(\mathrm{v} / \mathrm{v}) \mathrm{C}_{2}$. The growth was removed and used to inoculate $50 \mathrm{~mL}$. of GCB broth with supplements and grown as described above to late-logarithmic phase. The culture was diluted 10 -fold in $0.2 \times$ GCB broth and exposed to NHS [final concentration of $12.5 \%(\mathrm{v} / \mathrm{v})$ ] for $30 \mathrm{~min}$ and survivors were recovered by dilution plating onto GCB agar. This concentration of NHS was used because in preliminary experiments we found that use of a lower concentration of NHS resulted in inefficient killing and gave a high background of surviving gonococci that did not differ from parental strain F62 with respect to level of susceptibility to NHS (data not presented). After overnight growth, the colonies that survived in the presence of $12.5 \%$ NHS were pooled and reexposed to NHS as described above. This procedure was repeated for a third time and individual colonies were then screened along with parental strain F62 in the standard SBA. Ultimately, a mutant (WMS 100) that was less NHS-susceptible than parental strain F62 was identified. DNA was prepared from this mutant and used to transform F62 for Kan resistance and 10 individual transformants were screened in the NHS assay, all of which expressed the NHSresistance profile of the original mutant.

Ligation-polymerase chain reaction (PCR) andDNA sequencing was used to map the site of the transposon insertion as described previously (Pelicic et al., 2000). The oligonucleotide primers used for the ligationPCR were LMP1, LMP2, and IR1 (Pelicic et al., 2000). DNA sequencing was performed on a PCR product from a $\mathrm{PBAD}$ construct that contained the ligation-PCR product. This PCR product was sequenced using primer LGT19 (5'-GCGGTATTTGACCAAGGCTTC-3') and IR1 (Pelicic et al., 2000).

\section{CONSTRUCTION OF IgtA AND IptA INSERTIONAL MUTANTS}

Transformants of strain F62 bearing inactivated lgtA and/or lptA genes were constructed by transformation using donor

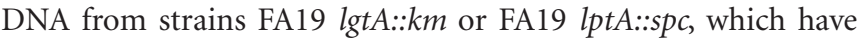


been described (Shafer et al., 2002; Lewis et al., 2009). The lgtA mutation were confirmed by PCR using oligonucleotide primers LGT5 (5'-CCGGCCTGATTCCCTCTTTAA-3') and LGT8 ( $5^{\prime}$-CCGGCAGTTCATACGCCGCT-3'). The lptA mutation was confirmed using LPTA4 (5'-GGCGGAAGGCTTTGGAAAAC-3') and LPTA5 (5'-GGCGAGTGCCTCGACA ATAT- ${ }^{\prime}$ ).

\section{COMPLEMENTATION ANALYSIS}

The pGCC4 complementation vector described previously (Skaar et al., 2002) was used for complementation of the lgtD:: $\mathrm{km}$ mutation generated by Himar I mariner mutagenesis. lgtD was PCR-amplified from genomic DNA using oligonucletotide primers that contained a PacI site (LGT15PAC; 5'-TTAATTAAGCCGTCTGAAGCCT-3'; restriction site is underlined) or PmeI site (LGT16Pme; $5^{\prime}$-GTTTAAACGCGGAACGGAAGC- ${ }^{\prime}$ ) and cloned into Pacl-PmeI digested pGCC4. The plasmid construct, which contains an IPTGinducible lacZ promoter (Skaar et al., 2002) was purified and used to transform strain WMS 100 (see Table 1) for resistance to chloramphenicol $(1 \mu \mathrm{g} / \mathrm{mL})$. The insert, positioned between the $l c t P$ and aspC Chromosomal sites (Skaar et al., 2002; Folster et al., 2009), was verified by PCR using primers LGT23 (5'-CGGCTGGATTGAGAAAATCGT-3') and LCTP (5'-GCGCGATCGGTG CGTTCT-3'). Expression of the insert was performed by growing the complemented strain (WMS 101) in GCB broth or on GCB agar containing $1 \mathrm{mMIPTG}$ as described previously (Folster et al., 2009). RT-PCR (Folster et al., 2009) was performed to confirm expression of the ectopically expressed $\lg t D$ gene in WMS 101. The lptA::spc mutation was complemented using the previously described (Lewis et al., 2009) pGCC4 construct that contained the wild type gene.

\section{LOS CHEMICAL ANALYSES}

Lipooligosaccharide produced by gonococcal strains were visualized by subjecting proteinase $\mathrm{K}$ digests of whole cell lysates to Tricine SDS-PAGE (Lesse et al., 1990) followed by silver staining (Tsai and Frasch, 1982), or Western immunoblotting (Towbin et al., 1979) using anti-LOS monoclonal antibodies 4C4,3F11, or 1-1-M as described (Gotschlich, 1994; Shafer et al., 2002), which recognize epitopes in the $\alpha$-chain of the core oligosaccharide.

Lipooligosaccharide was purified from $12 \mathrm{~L}$ batch cultures of strains grown in GCB broth with defined supplements I and II under 3.8\% $\mathrm{CO}_{2}$ as described (Lewis et al., 2009). In order to remove contaminating phospholipids the dried bacterial pellets

\begin{tabular}{llll}
\hline \multicolumn{4}{l}{ Table $\mathbf{1}$ | Description and susceptibility of strains to NHS and PB. } \\
\hline Strain & Genotype & $\begin{array}{l}\text { \% Survival in } \\
\mathbf{1 2 . 5} \% \text { NHS }\end{array}$ & PB MIC $(\boldsymbol{\mu g} / \mathbf{m L})$ \\
& & $2.15( \pm 1.24)$ & 50 \\
\hline F62 & Wild type & $51.8( \pm 18.1)^{*}$ & 50 \\
WMS 100 & F62 lgtD ::km & $1.65( \pm 1.45)^{* *}$ & 50 \\
WMS 101 & WMS 100 lgtD+ & $87.2( \pm 7.05)^{*}$ & 50 \\
WMS 102 & F62 lgtA::km & $2.37( \pm 2.17)^{* *}$ & 0.2 \\
WMS 103 & WMS 100 lptA::spc & 0.2 \\
WMS 104 & F62 lptA::spc & ND & 50 \\
WMS 105 & WMS 104 lptA & ND &
\end{tabular}

${ }^{*} p<0.001$ compared to F62; * $p<0.001$ compared WMS 100. $N D$, not determined. were extracted with a 9:1 ethanol-water mixture and then lyophilized. The oligosaccharides (OSs) were released from the LOS by mild acid hydrolysis $\left[1 \%(\mathrm{v} / \mathrm{v})\right.$ acetic acid at $100^{\circ} \mathrm{C}$ for $\left.2 \mathrm{~h}\right]$. The lipid A portions were precipitated by low-speed centrifugation, and supernatants containing the OS fraction were lyophilized and used for chemical and structural analyses as described (Shafer et al., 2002; Lewis et al., 2009). The OSs were treated with $48 \%$ hydrogen fluoride (HF) at $4^{\circ} \mathrm{C}$ for $48 \mathrm{~h}$ to remove any phosphate groups; e.g., phosphate or PEA. The HF-treated material was neutralized by ice-cold ammonium hydroxide solution (approximately $30 \%$ ) on an ice-water bath. The neutralized material was desalted by gel permeation chromatography using fine grade Bio-Gel P2 (Bio-Rad).

Glycosyl compositional analysis was performed by gas chromatography-mass spectrometry (GLC-MS) of trimethylsilyl (TMS) methyl glycosides with myoinositol used as an internal standard (York et al., 1985). The samples were hydrolyzed with methanolic $1 \mathrm{M} \mathrm{HCl}$ at $80^{\circ} \mathrm{C}$ for $18 \mathrm{~h}$. The released monosaccharides were dried under a stream of dry air and $\mathrm{N}$-acetylated with 3:1:1 methanol/pyridine/acetic anhydride $(\mathrm{v} / \mathrm{v} / \mathrm{v})$ at $100^{\circ} \mathrm{C}$ for $1 \mathrm{~h}$. After cooling, samples were dried-down and trimethylsilylated with Tri-sil reagent (Pierce) for $30 \mathrm{~min}$ at $80^{\circ} \mathrm{C}$. The resulting TMS derivatives were analyzed by GLC-MS, on Hewlett-Packard HP5890/ HP5970 MSD gas chromatograph/mass spectrometer equipped with Supelco DB-1 fused silica capillary column $(30 \mathrm{~m} \times 0.25 \mathrm{~mm}$ I.D.) with helium as the carrier gas.

Linkage analyses were carried out by the slurry $\mathrm{NaOH}$ method modified from that of Ciucanu and Kerek (1984). Samples were dissolved in $0.5 \mathrm{~mL}$ dimethyl sulfoxide (DMSO) by stirring overnight at room temperature under a $\mathrm{N}_{2}$ atmosphere. After dissolution, a freshly prepared slurry of $\mathrm{NaOH}$ in DMSO was added $(0.5 \mathrm{~mL})$ and the reaction mixture was stirred for $2 \mathrm{~h}$ at room temperature. Methylation was performed by the sequential addition of iodomethane $(250 \mu \mathrm{L}$ followed by $100 \mu \mathrm{L})$ at 30 min intervals. The permethylated monosaccharide was extracted into the organic phase after partitioning the reaction mixture between water and chloroform. The organic phase was then removed by evaporation under a stream of $\mathrm{N}_{2}$. The permethylated $\mathrm{OS}$ was hydrolyzed with $4 \mathrm{M}$ TFA $\left(100^{\circ} \mathrm{C}, 6 \mathrm{~h}\right)$, reduced with $\mathrm{NaBH}_{4}$, acetylated and the resulting partially methylated alditol acetates (PMAAs) were dissolved in dichloromethane and analyzed by GLC/MS using an HP-1 (from Hewlett-Packard) capillary column $(25 \mathrm{~m} \times 0.25 \mathrm{~mm})$.

Oligosaccharides were analyzed by matrix-assisted laser desorption ionization-time of flight mass spectrometry (MALDI-TOF MS) using a 4700 Proteomics Analyzer instrument (Applied Biosystems). The OS samples were dissolved in water $(1 \mu \mathrm{g} / \mu \mathrm{L})$, mixed in a $1: 1$ $(\mathrm{v} / \mathrm{v})$ ratio with $0.5 \mathrm{M} 2,5$-dihydroxybenzoic acid (DHB) in methanol matrix solution, and spotted on a stainless steel MALDI plate. Spectra were acquired in both the positive and negative acquisition modes. The acceleration voltage was set to $20 \mathrm{kV}$ and data were acquired in the reflectron mode with a 200-ms delay.

\section{RESULTS \\ ISOLATION OF A Himar IINSERTION MUTANT OF STRAIN F62 WITH DECREASED NHS-SUSCEPTIBILITY}

We employed strain F62 in this investigation for a number of important reasons. First, it was originally employed in the early 1960s for the development of a gonococcal typing scheme that 
was based on the state of piliation (Kellogg et al., 1963; piliated T1 and T2 vs. non-piliated T3 and T4 colony types). Second, it was employed in human experimental infection studies that identified pili as being important for establishing a gonococcal infection in male volunteers (Kellogg et al., 1968). Third, the LOS species it naturally produces have been defined (Yamasaki et al., 1991). Finally, since it is highly sensitive to killing by NHS, which is mediated by an antibody (natural IgM)- and CCP-dependent mechanism (Shafer et al., 1982), we reasoned that the killing capacity of NHS would be sufficient to use it as a selective pressure when screening the transposon library.

In order to isolate stable transposon mutants of strain F62 in the Himar I mariner library bank that expressed decreased susceptibility to NHS, the entire bank was grown in batch culture and exposed to $12.5 \%(\mathrm{v} / \mathrm{v})$ serum. This process was repeated twice and ultimately a transposon mutant (WMS 100) expressing decreased susceptibility to NHS (average of 51.8\% survival), compared to parent strain F62 (average of $2.15 \%$ survival), was identified (Table 1). A randomly picked Himar I mutant was used as a control in the NHS bactericidal assay and it was as NHS-susceptible as parent strain F62 (data not presented) indicating that the possession of the transposon per se was not responsible to the elevated NHSresistance displayed by WMS 100 .

In order to locate the site of the transposon insertion in WMS 100, we employed ligation-PCR and DNA sequencing (Pelicic et al., 2000). These procedures mapped the transposon insertion to the $5^{\prime}$-end of the lgtD coding sequence between nucleotide positions 61 and 62 (data not presented). The lgtD gene is part of a five gene operon $(\lg t A B C D E)$ and it, as well as $\operatorname{lgt} A$ and $\operatorname{lgt} C$, are phase variable due to polymeric repeat sequences (Gotschlich, 1994; Yang and Gotschlich, 1996; Shafer et al., 2002). Due to the phase variable property of $\operatorname{lgt} D$, it was not clear if the observed NHS-resistance property of WMS 100 was due to the transposon insertion or spontaneous mutation in other genes. To determine if transposon insertion within $\lg t D$ was responsible for the increased NHS-resistance property of strain WMS 100, DNA from this mutant was used to transform parent strain F62 for $\operatorname{Kan}^{\mathrm{R}}$. Analysis of 10 individual transformants revealed that all expressed decreased susceptibility to NHS (data not presented) similar to WMS 100, indicating that the serum susceptibility phenotype was linked to the transposon insertion. Further verification that the transposon insertion in $\operatorname{lgt} D$ in WMS 100 was responsible for its decreased NHS-susceptibility phenotype was obtained by complementation analysis in which the wild type $\lg t D$ gene from parental strain F62 was expressed ectopically from the $l c t P-a s p C$ region under the control of the lac promoter (Skaar et al., 2002). With complemented strain WMS 101, we found that expression of the wild type $\operatorname{lgtD}$ resulted in an NHSsusceptible phenotype (average of $1.65 \%$ survival) similar to strain F62 (Table 1).

\section{CHARACTERIZATION OF LOS DUE TO TRANSPOSON INSERTION IN IgtD}

The major LOS species produced by strains F62 have been structurally characterized (Yamasaki et al., 1991). Herein, we determined whether the LOS species produced by strain F62 would differ from WMS 100 and if those produced by complemented strain WMS 101 would resemble that of F62. We first identified the major
LOS species produced by these strains by subjecting proteinase $\mathrm{K}$ digests of their whole cell lysates to SDS-PAGE. Silver staining of the gels revealed that F62 and complemented strain WMS 101 produced two in predominant LOS species (data not presented but summarized in Table 2) that migrated at 4.5 and $4.8 \mathrm{kDa}$. In contrast, mutant strain WMS 100 produced a single LOS species that migrated at $4.5 \mathrm{kDa}$. The $4.5-\mathrm{kDa}$ species was reactive with mAb 3F11 (which recognizes Gal-GlcNAc-Gal-Glc-HepI), while the $4.8-\mathrm{kDa}$ species was reactive with $\mathrm{mAb} 1-1-\mathrm{M}$ (which recognizes GalNAc-Gal-GlcNAc-Gal-Glc-HepI; Table 2); neither of these species bound $\mathrm{mAb} 4 \mathrm{C} 4$, which recognizes Gal-Glc-HepI in the 3.6-kDa species previously linked to stable NHS-resistance in strain FA19 (Shafer et al., 2002). Based on earlier LOS-epitope mapping experiments, the electrophoretic mobility of the LOS species, the reported structure of F62 LOS species (Yamasaki et al., 1991) and the deduced $N$-acetylgalactosamine transferase activity of LgtD (Gotschlich, 1994), we hypothesized that the LOS produced by F62 and WMS 100 would differ by the absence of a terminal $N$-acetylgalactosamine in the $\alpha$-chain. Indeed, chemical and structural analyses (Figure 1; Table 3) of the LOS produced by F62 and WMS 100 confirmed this inference.

For structural analysis the OSs from F62 and WMS 100 LOSs were treated with aqueous HF to remove any phosphate groups, as described in the Methods, in order to facilitate glycosyl composition and linkage analyses as well as MS analysis of the OSs. Glycosyl composition analysis of the HF-treated OS fraction from wild type F62 showed the presence of glucose (Glc), galactose (Gal), N-acetylglucosamine (GlcNAc), N-acetylgalactosamine (GalNAc), heptose (Hep), and 3-deoxy-D-manno-2-octulosonic acid (Kdo). Composition analysis of the HF-treated OS from the lgtD::km mutant strain (WMS 100) showed the same glycosyl components with the exception that GalNAc was not detected. Glycosyl linkage analysis showed that the F62 OS fraction contained terminally linked GalNAc ( $\mathrm{t}-\mathrm{GalNAc}$ ), $\mathrm{t}$-Gal, $\mathrm{t}$-GlcNAc, 4-linked GlcNAc (4-GlcNAc), 4-Glc, 3-Gal, 2-Hep, and 3,4-Hep. The OS from mutant strain WMS 100 contained all of the same residues except that it was completely devoid of $\mathrm{t}-\mathrm{GalNAc}$ and the t-Gal: 3-Gal ratio was increased.

The HF-treated OS fraction from each strain was subjected to MALDI-TOF MS analysis and the results are shown in Figure 1 and in Table 3. The wild type F62 OS fraction shows two ion clusters

Table 2 | Lipooligosaccharide species produced by gonococcal strains.

\begin{tabular}{lllll}
\hline Strain & $\begin{array}{l}\text { LOS } \\
\text { species (kDa) }\end{array}$ & \multicolumn{3}{c}{$\begin{array}{c}\text { Reactivity with } \\
\text { mAbs }\end{array}$} \\
\cline { 3 - 5 } & & $\mathbf{3 F 1 1}$ & $\mathbf{1 - 1 - M}$ & $\mathbf{4 C 4}$ \\
\hline F62 & 4.5 & + & - & - \\
& 4.8 & - & + & - \\
WMS 100 & 4.5 & + & - & - \\
WMS 101 & 4.5 & + & - & - \\
& 4.8 & - & + & - \\
WMS 102 & 3.6 & - & - & + \\
\hline
\end{tabular}

Lipooligosaccharide species are given in kilodaltons ( $k D a)$ as determined by Tricine SDS-PAGE analysis. 


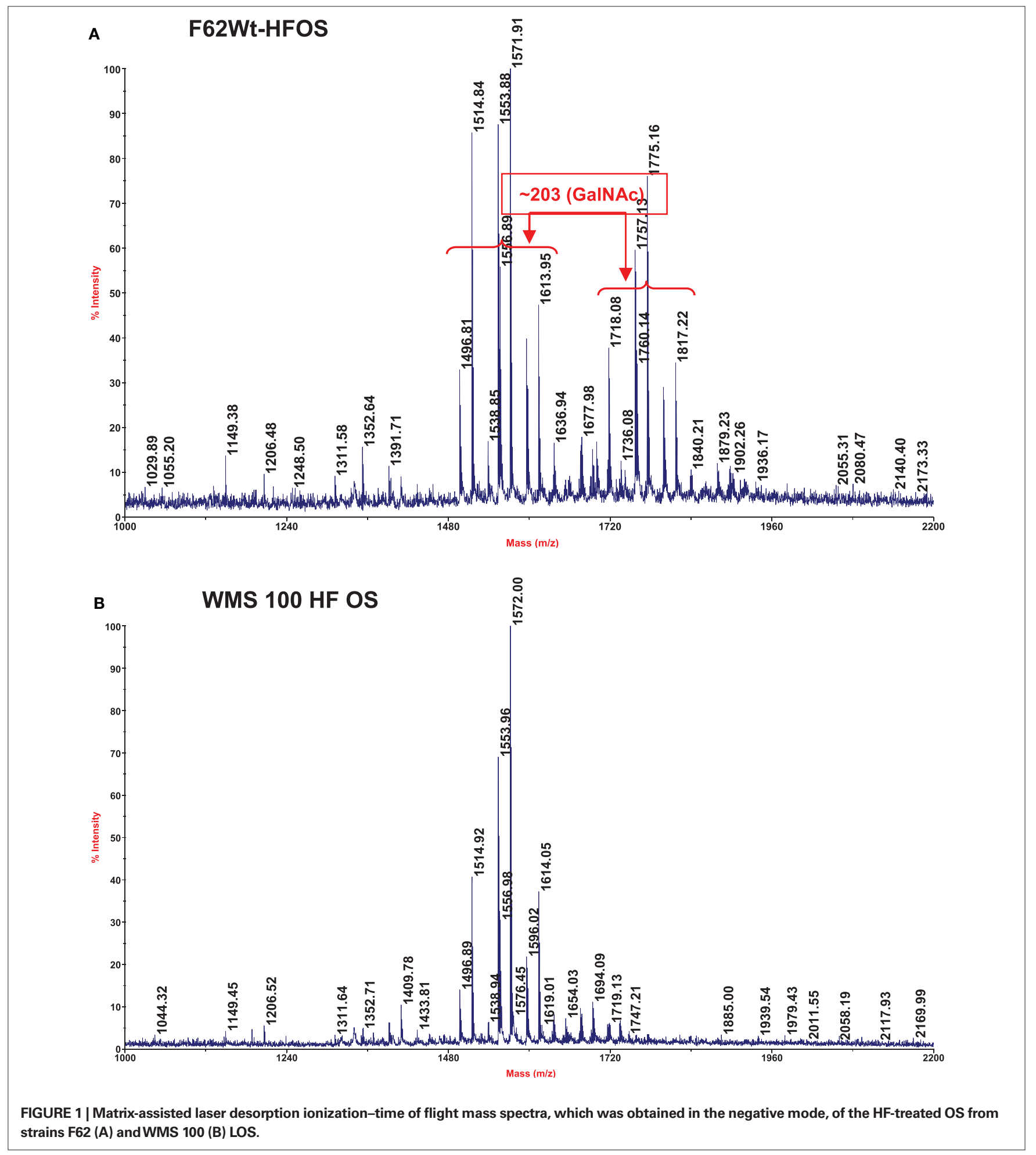

with the higher molecular weight cluster differing from the lower molecular weight cluster by 203 mass units, the mass of a HexNAc residue (Figure 1). Both ion clusters also contained masses that differed by 57 mass units; the mass of glycine (Gly) indicated that Gly-containing structures with and without a HexNAc residue were present. The WMS 100 mutant OS fraction showed a single ion cluster that contains the same ions as observed for the lower molecular weight ions present in the wild type $\mathrm{F} 62 \mathrm{OS}$ fraction; i.e., only those ions that lack a HexNAc residue are present in the WMS 100 mutant OS. The proposed compositions, based on the glycosyl residue analysis described above, for the observed ions are shown in Table 3 together with the observed and calculated $\mathrm{m} / \mathrm{z}$ values. 
Table 3 | lons, [M-H]', observed on mass spectrometric analysis for the HF-treated oligosaccharides from wild type F62 and mutant WMS 100 lipooligosaccharides.

\begin{tabular}{|c|c|c|c|c|}
\hline Obs. mass & Cal. mass & $\begin{array}{l}\text { Proposed } \\
\text { composition }\end{array}$ & F62 & WMS 100 \\
\hline 1556.9 & 1556.5 & $\begin{array}{l}\mathrm{Gal}_{2} \mathrm{GlcGlcNAc}_{2} \\
\mathrm{Hep}_{2} \cdot \mathrm{KdoOAc}\end{array}$ & + & + \\
\hline 1614.0 & 1613.6 & $\begin{array}{l}\mathrm{Gal}_{2} \mathrm{GlcGlcNAc}_{2} \\
\mathrm{Hep}_{2} \cdot \mathrm{KdoOAcGly}\end{array}$ & + & + \\
\hline 1514.8 & 1514.4 & $\mathrm{Gal}_{2} \mathrm{GlcGlcNAc}_{2} \mathrm{Hep}_{2} \cdot \mathrm{Kdo}$ & + & + \\
\hline 1496.8 & 1496.4 & $\begin{array}{l}\mathrm{Gal}_{2} \mathrm{GlcGlcNAc}_{2} \mathrm{Hep}_{2} \\
\text { Kdo (anhydro) }\end{array}$ & + & + \\
\hline 1571.9 & 1571.5 & $\mathrm{Gal}_{2} \mathrm{GlcGlcNAc}_{2} \mathrm{Hep}_{2}$. KdoGly & + & + \\
\hline 1553.9 & 1553.5 & $\begin{array}{l}\mathrm{Gal}_{2} \mathrm{GlcGl}_{\mathrm{cNAc}} \mathrm{Hep}_{2} \\
\text { KdoGly (anhydro) }\end{array}$ & + & + \\
\hline 1760.1 & 1759.7 & $\begin{array}{l}\text { GalNAcGal }_{2} \text { GlcGlcNAc }_{2} \\
\mathrm{Hep}_{2} \mathrm{KdoOAc}\end{array}$ & + & - \\
\hline 1817.2 & 1816.8 & $\begin{array}{l}\text { GalNAcGal }_{2} \text { IlcGlcNAc }_{2} \\
\mathrm{Hep}_{2} \mathrm{KdoOAcGly}\end{array}$ & + & - \\
\hline 1718.1 & 1717.7 & $\begin{array}{l}\text { GalNAcGal } \mathrm{GlcGl}_{2} \\
\mathrm{NAc}_{2} \mathrm{Hep}_{2} \mathrm{Kdo}\end{array}$ & + & - \\
\hline 1775.2 & 1774.7 & $\begin{array}{l}\text { GalNAcGal }_{2} \text { GlcGlcNAc }_{2} \\
\mathrm{Hep}_{2} \mathrm{KdoGly}\end{array}$ & + & - \\
\hline 1757.1 & 1756.7 & $\begin{array}{l}\text { GalNAcGal }_{2} \text { GlcGlcNAc }_{2} \\
\mathrm{Hep}_{2} \mathrm{KdoGly} \text { (anhydro) }\end{array}$ & + & - \\
\hline
\end{tabular}

Shown are the observed (obs.) and calculated (cal.) masses for each OS species in strains.

F62 and WMS 100 with presence (+) or absence (-) of species designated.

The complete structures of the OSs for the F62 LOS have been reported (Yamasaki et al., 1991). These structures are shown in Figure 2. Our glycosyl composition, linkage, and MS results are consistent with this report by Yamasaki et al. (1991), which shows that the F62 LOS contains the two OSs shown in Figure 2, one with a t-GalNAc residue and one that lacks this residue. The lack of the $\mathrm{t}$-GalNAc residue in the LOS from mutant WMS 100, as well as the MS, glycosyl composition, and linkage analyses is consistent with the conclusion that this LOS contains the OS structure shown in Figure 2 that lacks the GalNAc residue and confirms that the $\operatorname{lgt} D$ gene encodes the GalNAc transferase.

\section{PRODUCTION OF A 3.6-kDa LOS RESULTS IN INCREASED NHS- RESISTANCE IN STRAIN F62}

Since our earlier work (Shafer et al., 2002) with the naturally NHSresistant strain FA19 revealed that production of a 3.6-kDa LOS species with the LOS $\alpha$-chain, which consists of Gal-Glc-HepI, was important in NHS-resistance, we next asked if production of a similarly truncated LOS by normally highly NHS-sensitive F62 would result in a level of NHS-resistance greater than that expressed by $\lg t D$ mutant strain WMS 100 . We addressed this matter because our screening of the transposon library for NHSresistant variants did not identify an insertion in $\operatorname{lgt} A$, which based on our earlier work with strain FA19 (Shafer et al., 2002), is known to be important in NHS-resistance. In order to test the

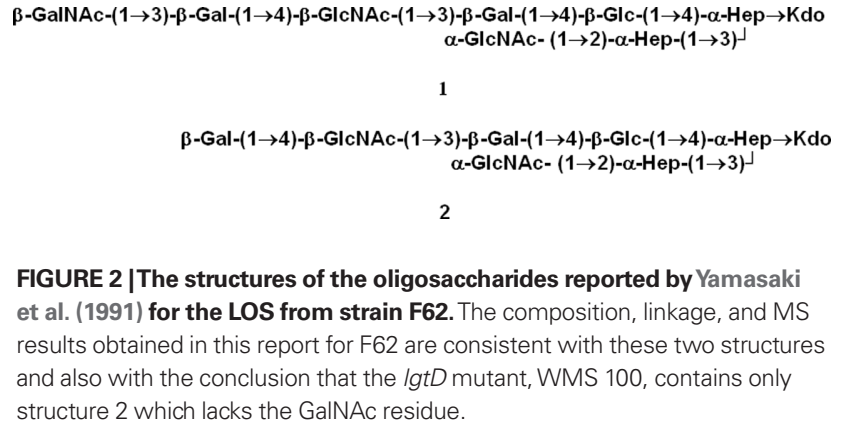

importance of $\lg t A$, we introduced a non-polar $\operatorname{lgtA}:: \mathrm{km}$ mutation into strain F62 since LgtA is glycosyltransferase that adds GlcNAc to the terminal galactose in the $\alpha$-chain of the $3.6-\mathrm{kDa}$ LOS (Gotschlich, 1994; Shafer et al., 2002). A resulting lgtA::km transformant (WMS 102) was found to produce a single LOS species $(3.6 \mathrm{kDa})$ that was reactive with mAb 4C4 (Table 2), but not 3F11 or 1-1-M. Importantly, WMS 102 expressed a level of NHS-resistance (average of $87.2 \%$ survival) that was substantially greater than that seen in the lgtD mutant WMS 100 (Table 1) or parental strain F62. However, incubation of WMS 102 (or WMS $100)$ in higher concentrations (>25\%) of NHS resulted in less survival $(<1 \%)$ of gonococci, while a naturally NHS-resistant strain (FA19) showed complete resistance ( $>100 \%$ survival). Thus, truncation of the LOS $\alpha$-chain in F62 is not sufficient for complete NHS-resistance at higher serum levels presumably because it still possesses a Por1B protein as opposed to the Por1A produced by FA19 (Shafer et al., 2002). Nevertheless, since complement components can be at reduced levels at mucosal surfaces (Rice, 1989), phase-variants of naturally NHS-sensitive gonococci that produce truncated LOS species due to spontaneous mutations in $\operatorname{lgt} A$ or $\lg t D$ would have a survival advantage against this mechanism of innate host defense.

\section{LOSS OF PEA MODIFICATION OF LIPID A INCREASES NHS- SUSCEPTIBILITY OF GONOCOCCI}

Having determined that the length of the LOS $\alpha$-chain is important in determining levels of NHS-susceptibility expressed by strain F62, we next examined whether the structure of its lipid A can also contribute to resistance. In this respect, we recently showed (Lewis et al., 2009) that loss of 4' PEA from lipid A due to a null mutation in $l p t A$ could render normally NHS-resistant strain FA19 highly susceptible to NHS through a classical pathway-dependent mechanism. In order to test if such PEA modification of the lipid A was important in the ability of WMS 100 to express decreased susceptibility to NHS, we created an lptA::spc transformant (see strain WMS 103). The results (Table 1) showed that WMS 103 was substantially more NHS-susceptible (average of $1.3 \%$ survival) than parent strain WMS 100. In fact, the lptA mutation negated the NHS-resistance profile afforded by the lgtD mutation. Thus, from our studies on the Himar I transposon mutant strain WMS 100, we conclude that both the length of the $\alpha$-chain of the core oligosaccharide and 4' lipid A PEA are important LOS structures that can determine the extent of gonococcal susceptibility to NHS. 
LOS STRUCTURE AND CAP SUSCEPTIBILITY EXPRESSED BY GONOCOCCI Cationic antimicrobial peptides are on the first line of innate host defense and can kill microbes directly by damaging their membranes or inhibiting intracellular processes (Brogden, 2005). They also indirectly kill microbes by virtue of their immunostimulatory, proinflammatory activities (Easton et al., 2009). Substantial evidence exists that alterations in lipid A structure can modulate levels of bacterial susceptibility to CAPs (Lee et al., 2004; Lewis et al., 2009). Accordingly, we tested if the LOS mutants described above would differ from parental strain F62 in their susceptibility to PMB, a model CAP. Although truncation of the LOS $\alpha$-chain did not greatly influence the susceptibility of strain F62 to PMB, the presence of the lptA::spc mutation rendered strain F62 hypersusceptible to PMB (250-fold compared to parent strain F62; Table 1). Complementation of the mutant with the wild type lptA gene expressed from the lacZ promoter enhanced PMB-resistance by $>60$-fold (Table $\mathbf{1}$ ).

\section{DISCUSSION}

A long-standing interest in gonococcal research has been to identify and characterize the molecular mechanisms that explain how this strict human pathogen can resist innate and adaptive immunity systems. We have been interested in how the gonococcus can evade killing by the complement system and host defense peptides (e.g., CAPs). In previous studies, we and others identified genes of interest and constructed directed mutations for subsequent testing in antimicrobial assays. While this has proven to be a useful strategy, it is in fact biased for a select panel of genes. We have wanted an unbiased approach so that the killing power of host defense systems could be used to directly identify such mutants. To facilitate this effort, we employed the Himar I mariner in vitro transposon mutagenesis system for isolation of mutants of $N$. gonorrhoeae that manifest decreased susceptibility to complement-mediated killing by NHS. Through this strategy and with follow-up studies using gene-directed mutagenesis, we found that historically important strain F62 has LOS-dependent susceptibility to two components of innate host defense: the bactericidal action of NHS and CAPs. In this respect, the results presented herein and elsewhere (Shafer et al., 2002; Lewis et al., 2009) show that changes in the extension of the LOS $\alpha$-chain, which can occur at high frequencies due to phase variable expression of genes in the lgtABCDE operon (Gotschlich, 1994; Yang and Gotschlich, 1996), can determine levels of gonococcal susceptibility to NHS (Shafer et al., 2002) while PEA decoration of lipid A is important in CAP-resistance (Lewis et al., 2009).

Normal human serum-resistance in gonococci has been linked to the serovar of the major outer membrane porin (Por1A vs. Por 1B) produced. Por 1B-producing gonococci are more frequently killed by NHS than Por1A strains. There is growing evidence, however, that the structure of LOS is important in NHS-resistance (Shafer et al., 2002; Ram et al., 2003) and other pathogenic steps used by

\section{REFERENCES}

Alexander, H, Rasmussen, A., and Stojiljkovic, I. (2006). Identification of Neisseria meningitidislociinvolved in the modulation of phase variation frequencies. Infect. Immun. 72, 6743-6747.
Apicella, M. A., Shero, M., Jarvis, G. A., Griffiss, J. M., Mandrell, R. E., and Schneider, H. (1987). Phenotypic variation in epitope expression of the Neisseria gonorrhoeaelipooligosaccharide. Infect. Immun. 55, 1755-1761.

gonococci during infection (van Putten, 1993), including bacterial attachment to host cells, stimulation of inflammatory responses and resistance to CAPs. Since an individual strain (and colony of that strain) can produce multiple LOS species due largely to the presence of phase variable expression of LOS biosynthesis genes (Apicella et al., 1987; Schneider et al., 1991; Preston et al., 1996; Burch et al., 1997; Tong et al., 2002), it has been difficult to ascribe with certainty an LOS chemotype with a particular pathogenic property. The results presented herein and that of earlier work on the role of the LOS $\alpha$-chain and its extension by glycosyltransferases in determining whether gonococci can be killed by NHS (Shafer et al., 2002) demonstrate that extension of this chain past Gal-Glc-HepI-Kdo can result in NHS-susceptibility. The enhanced NHS-susceptibility observed in strains producing an extended LOS $\alpha$-chain is likely due to natural IgM antibodies directed against epitopes present in such structures (Gotschlich, 1994). The NHSresistance property displayed by transposon mutant WMS 100 may reflect a decrease in LOS affinity for such antibodies due to loss of the terminal $\mathrm{N}$-acetyl galactosamine present in parental strain F62. Since $\lg t D$ is naturally phase variable, those gonococci having a phase-off gene might have a fitness advantage over those with a phase-on gene since they would have an enhanced ability to escape NHS-killing mediated by the CCP at sites where complement levels are limited. Under these circumstances, naturally occurring phaseoff lgt $A$ or $\lg t D$ mutants of Por 1B-producing strains would have an advantage over parent PorIB strains with phase-on sequences of these genes. NHS-resistance expressed by gonococci also requires PEA modification of gonococcal lipid A and this decoration also enhances resistance to CAPs. We previously proposed (Lewis et al., 2009) that PEA decoration of lipid A influences the efficacy of complement regulatory protein $\mathrm{C} 4 \mathrm{BP}$ binding to the surface of gonococci. C4BP binds to most gonococcal PorlA and select Por1B molecules. With respect to CAPs, loss of PEA from lipid A would enhance ionic interaction of positively charged groups in CAPs with phosphates at the 1 and $4^{\prime}$ positions of lipid A that become available, resulting in enhanced susceptibility of gonococci to CAPs. Using a murine model of vaginal infection, we are now testing whether mutations that impact LOS core oligosaccharide or lipid A structure and influence levels of resistance to mediators of innate host defense impact the in vivo fitness and survival of gonococci during infection.

\section{ACKNOWLEDGMENTS}

We thank Lane Pucko for help in manuscript preparation. This work was supported by a VA Merit grant (William M. Shafer), NIH grant AI0311496-20 (P. F. Sparling, University of North Carolina) and Department of Energy grant DE-FG02-09ER20097 to the Complex Carbohydrate Center of the University of Georgia. William M. Shafer is the recipient of a Senior Research Career Scientist Award from the VA Medical Research Service.

Brogden, K.A. (2005). Antimicrobial peptides: pore formers or metabolic inhibitors in bacteria. Nat. Rev. Microbiol. 3, 238-250.

Burch, C. L., Danaher, R. J., and Stein, D. C. (1997). Antigenic variation in
Neisseria gonorrhoeae: production of multiple lipooligosaccharides. J. Bacteriol. 179, 982-986.

Cannon, J. G., Buchanan, T. M., and Sparling, P. F. (1983). Confirmation of association of protein I serotype of 
Neisseria gonorrhoeae with the ability to cause disseminated infection. Infect. Immun. 40, 816-819.

Ciucanu, I., and Kerek, F. (1984). A simple and rapid method for the permethylation of carbohydrates. Carbohydr. Res. 131, 209-217.

Easton, D. M., Nijnik, A., Mayer, M. L., and Hancock, R. E. W. (2009). Potential of immunomodulatory host defense peptides as novel anti-infectives. Trends Biotechnol. 10, 582-590.

Folster, J. P., Johnson, P. J. T., Jackson, L., Dhulipala, V., Dyer, D. W., and Shafer, W. M. (2009). MtrR modulates $r p o H$ expression and levels of antimicrobial resistance in Neisseria gonorrhoeae. J. Bacteriol. 191, 287-297.

Gotschlich, E. C. (1994). Genetic locus for the biosynthesis of the variable portion of Neisseria gonorrhoeae lipooligosaccharide. J. Exp. Med. 180, 2181-2190.

Gunn, J., and Stein, D. C. (1996). Use of a non-selective transformation technique to construct a multiple restriction/modification-deletion mutant of Neisseria gonorrhoeae. Mol. Gen. Genet. 251, 509-517.

Kellogg, D. S., Cohen, I. R., Norins, L. C., Schroeter, A. L., and Reising, G. (1968). Neisseria gonorrhoeae II: colony variation and pathogenicity during 35 months in vitro. J. Bacteriol. 96, 596-605.

Kellogg, D. S., Peacock, W. L., Deacon, W. E., Brown, L., and Pirkle, C. I. (1963). Neisseria gonorrhoeae I: virulence genetically linked to clonal variation. J. Bacteriol. 85, 1274-1279.

Lee, H., Hsu, F. F., and Groisman, E. A. (2004). The PmrA-regulated pmrC gene mediates phosphoethanolamine modification of lipid A and polymyxin B resistance in Salmonella enterica. J. Bacteriol. 186, 4124-4133.

Lesse, A. J., Campagnari, A. A., Bittner, W. E., and Apicella, M.A. (1990).Increased resolution of lipopolysaccharides and lipooligosaccharides utilizing tricinesodium dodecyl sulfate-polyacrylamide gel electrophoresis. J. Immunol. Methods 126, 109-117.

Lewis, L., Choudhury, B., Balthazar, J. T., Martin, L. E., Ram, S., Rice, P. A., Stephens, D.S., Carlson, R., and Shafer, W. M. (2009). Phosphoethanolamine substitution of lipid A and resistance of Neisseria gonorrhoeae to cationic antimicrobial peptides and complement-mediated killing by normal human serum. Infect. Immun. 77, 1112-1120.
Pelicic, V., Morelle, S., Lampe, D., and Nassif, X. (2000). Mutagenesis of Neisseria meningitidis by in vitro transposition of HimarI mariner. J. Bacteriol. 182, 5391-5398.

Preston, A., Mandrell, R. E., Gibson, B. E., and Apicella, M. A. (1996). The lipooligosaccharides of pathogenic Gram-negative bacteria. CRC Crit. Rev. Microbiol. 22, 139-180.

Ram, S. A., Cox, A. D., Wright, J. C., Vogel, S., Getzlaff, S., Boden, R., Li, J., Plested, J. S., Meri, S., Gulati, S., Stein, D. C., Richards, J. C., Moxon, E. R., and Rice, P. A. (2003). Neisserial lipooligosaccharide is a target for complement component C4b. Inner core phosphoethanolamine residues define $\mathrm{C} 4 \mathrm{~b}$ linkage specificity. J. Biol. Chem. 280, 50853-50862.

Ram, S., Cullinane, M., Blom, A. M., Gulati, S., McQuillen, D. P., Monks, B. G., O’Connell, C., Boden, R., Elkins, C., Pangborn, M. K., Dahlback, B., and Rice, P. A. (2001). Binding of C4b-binding protein to porin: a molecular mechanism of serum resistance in Neisseria gonorrhoeae. J. Exp. Med. 193, 281-295.

Ram, S., McQuillen, D., Gulati, S., Elkins, C., Pangburn, M., and Rice, P. (1998). Binding of complement $\mathrm{H}$ to loop 5 of porin protein 1A: a molecular mechanism of serum resistance of nonsialylated N. gonorrhoeae. J. Exp. Med. 188, 671-680.

Rice, P. A. (1989). Molecular basis for serum resistance in Neisseria gonorrhoeae. Clin. Microbiol. Rev. 2, S112-S117.

Rice, P.A., McCormack, W.M., and Kasper, D. L. (1980). Natural serum bactericidal activity against Neisseria gonorrhoeae isolated from disseminated, locally invasive and uncomplicated disease. J. Immunol. 124, 2105-2109.

Schneider, H., Griffiss, J. M., Boslego, J., Hitchcock, P. J., Zahos, K. M., and Apicella, M. A. (1991). Expression of paragloboside-like lipooligosaccharides may be a necessary component of gonococcal pathogenesis in men. J. Exp. Med. 174, 1601-1605.

Schneider, H., Griffiss, J. M., Mandrell, J. E., and Jarvis, G.A. (1985). Elaboration of a 3.6 kilodalton lipooligosaccharide, antibody against which is absent from human serum, is associated with serum resistance of Neisseria gonorrhoeae. Infect. Immun. 50, 672-677.

Schoolnik, G. K., Buchanan, T. M., and Holmes, K. K. (1976). Gonococci causing disseminated gonococcal infection are resistant to the bactericidal action of normal human serum. J. Clin. Invest. 58, 1163-1173.

Shafer, W. M., Datta, A., Kumar Kolli, V. S., Rahman, M. M., Balthazar, J. T., Martin, L. E., Veal, W. L., Stephens, D. S., and Carlson, R. W. (2002). Phase variable changes in genes lgtA and $\operatorname{lgt} C$ within the lgtABCDE operon of Neisseria gonorrhoeae can modulate gonococcal susceptibility to normal human serum. J. Endotoxin Res. 8, 47-58.

Shafer, W. M., Guymon, L. F., and Sparling, P. F. (1982). Identification of a new genetic site $\left(\mathrm{sac}^{-} \mathrm{3}^{+}\right)$in Neisseria gonorrhoeae that affects sensitivity to normal human serum. Infect. Immun. 35, 764-769.

Shafer, W. M., Joiner, K., Guymon, L. F., Cohen, M. S., and Sparling, P.F. (1984). Serum sensitivity of Neisseria gonorrhoeae: the role of lipopolysaccharide. J. Infect. Dis. 149, 175-183.

Shafer, W. M., Qu, X.-D., Waring, A. J., and Lehrer, R. I. (1998). Modulation of Neisseria gonorrhoeae susceptibility to vertebrate antibacterial peptides due to a member of the resistance/ nodulation/division efflux pump family. Proc. Natl. Acad. Sci. U.S.A. 95, 1829-1833.

Skaar, E. P., Lazio, M. P., and Seifert, H. S. (2002). Roles of the recJand recNgenes in homologous recombination and DNA repair in Neisseria gonorrhoeae. J. Bacteriol. 184, 919-927.

Southern, E. M. (1975). Detection of specific sequences among DNA fragments separated by gel electrophioresis. $J$. Mol. Biol. 98, 503-517.

Sparling, P. F., Tsai, J., and Cornelissen, C. N. (1990). Gonococci are survivors. Scand. J. Infect. Dis. S69, 125-136.

Tong, Y., Arking. D., Ye, S., Reinhold, B., Reinhold, V., and Stein, DC. (2002). Neisseria gonorrhoeae strain PID2 simultaneously expresses six chemically related lipooligosaccharide structures. Glycobiology 12, 523-533.

Towbin, H., Staehelin, T., and Gordon, J. (1979). Electrophoretic transfer of proteins from polyacrylamide gels to nitrocellulose sheets: procedures and applications. Proc. Natl. Acad. Sci. U.S.A. 76, 4350-4354.

Tsai, C.-M., and Frasch, C. E. (1982). A sensitive silver stain for detecting lipopolysaccharides in polyacrylamide gels. Anal. Biochem. 119, 115-119.

van Putten, J. P. M. (1993). Phase variation of lipopolysaccharide directs interconversion of invasive and immuno-resistant phenotypes of Neisseria gonorrhoeae. EMBO J. 12, 4043-4051.

Veal, W. L., Nicholas, R., and Shafer, W. M. (2002). Overexpression of the MtrC-MtrD-MtrE efflux pump due to an $m t r R$ mutation is required for chromosomally-mediated penicillin resistance in Neisseria gonorrhoeae. J. Bacteriol. 184, 5619-5624.

Yamasaki, R., Bacon, B. E., Nasholds, W., Schneider, H., and Griffiss, J. M. (1991). Structural determinations of oligosaccharides derived from lipooligosaccharides of Neisseria gonorrhoeae F62 by chemical, enzymatic and two-dimensional NMR methods. Biochemistry 30, 10566-10575.

Yang, Q. L., and Gotschlich, E. C. (1996). Variation of gonococcal lipooligosaccharide structures is due to alterations in poly-G tracts in lgt genes encoding glycosyl transferases. J. Exp. Med. 183, 323-327.

York, W. S., Darvill, A. G., McNeil, M., Stevenson, T. T., and Albersheim, P. (1985). Isolation and characterization of plant cell walls and cell wall components. Methods Enzymol. 118, 3-40.

Conflict of Interest Statement: The authors declare that the research was conducted in the absence of any commercial or financial relationships that could be construed as a potential conflict of interest.

Received: 12 January 2011; paper pending published: 02 February 2011; accepted: 04 February 2011; published online: 18 February 2011.

Citation: Balthazar JT, Gusa A, Martin LE, Choudhury B, Carlson R and Shafer WM (2011) Lipooligosaccharide structure is an important determinant in the resistance of Neisseria gonorrhoeae to antimicrobial agents of innate host defense. Front. Microbio. 2:30. doi: 10.3389/ fmicb.2011.00030

This article was submitted to Frontiers in Cellular and Infection Microbiology, a specialty of Frontiers in Microbiology.

Copyright (c) 2011 Balthazar, Gusa, Martin, Choudhury, Carlson and Shafer. This is an open-access article subject to an exclusive license agreement between the authors and Frontiers Media SA, which permits unrestricted use, distribution, and reproduction in any medium, provided the original authors and source are credited. 